\title{
Optimization of Packet Length for Two Way Relaying with Energy Harvesting
}

\author{
Ghassan Alnwaimi *,Hatem Boujemaa **, Kamran Arshad *** \\ (*) King Abdulaziz University, Kingdom of Saudi Arabia \\ $(* *)$ University of Carthage, Sup'Com, COSIM Laboratory, Tunisia \\ $(* * *)$ College of Engineering, Ajman University \\ galnwaimi@kau.edu.sa,boujemaa.hatem@supcom.tn, k.arshad@ajman.ac.ae
}

May 16, 2019

\begin{abstract}
In this article, we suggest optimizing packet length for two way relaying with energy harvesting. In the first transmission phase, two source nodes $N_{1}$ and $N_{2}$ are transmitting data to each others through a selected relay $R$. In the second phase, the selected relay will amplify the sum of the signals received signals from $N_{1}$ and $N_{2}$. The selected relay amplifies the received signals using the harvested energy from Radio Frequency (RF) signals transmitted by nodes $N_{1}$ and $N_{2}$. Finally, $N_{1}$ will remove, from the relay's signal, its own signal to be able to decode the symbol of $N_{2}$. Similarly, $N_{2}$ will remove, from the relay's signal, its own signal to be able to decode the symbol of $N_{1}$. We derive the outage probability, packet error probability and throughput at $N_{1}$ and $N_{2}$. We also optimize packet length to maximize the throughput at $N_{1}$ or $N_{2}$.
\end{abstract}

Index Terms : Cooperative systems, Optimal packet length, Rayleigh fading channels.

\section{Introduction}

In Two-Way Relaying (TWR), two nodes $N_{1}$ and $N_{2}$ simultaneously transmit data to each other using a selected relay [1-5]. The communication process contains two phases. In the first one, $N_{1}$ and $N_{2}$ transmit data to some relays. Each relay will receive the sum of signals transmitted by $N_{1}$ and $N_{2}$. In the second phase, a selected relay amplifies the received signal. Then, $N_{1}$ will remove, from the relay's signal, its own signal to be able to decode the symbol of $N_{2}$. Similarly, $N_{2}$ will remove, from the relay's signal, its own signal to be able to decode the symbol of $N_{1}$.

Two way relaying for Multiple Input Multiple Output (MIMO) systems has been considered in [1-5]. Receive and transmit diversity improves the performance of TWR. At the receiver, the best antenna can be selected (Selection Combining SC). The corresponding 
Signal to Noise Ratio (SNR) is the maximum of SNRs over all antennas. It is also possible to combine the signals of all antennas using Maximum Ratio Combining (MRC). The SNR will be the sum of all SNRs [1-5]. TWR with Energy Harvesting (EH) consists to use the Radio Frequency (RF) signal to charge the battery of nodes [6-10]. Relays with EH capabilities has been studied in [6-10]. In order to enhance the throughput especially at low SNRs, channel coding is required in TWR [11-13]. Secure two way relaying has been suggested in [14-20]. Security aspects of TWR should be studied to avoid data recovery by a malicious node.

The main contribution of the paper is to optimize packet length so that the throughput at node $N_{1}$ or $N_{2}$ is maximized. In all previous studies, a Fixed Packet Length (FPL) is used [1-20]. This is the first paper to suggest an Optimal Packet Length (OPL) for TWR with Energy Harvesting.

The system model is presented in section 2. Section 3 gives the Cumulative Distribution Function (CDF) of SNR. Section 4 derives the PEP while section 5 gives the expression of OPL. Some numerical results are given in section 6 . Conclusions are presented in section 7 .

\section{System model}

The system model is shown in Fig. 1. There are two nodes $N_{1}$ and $N_{2}$ communicating information to each other through a relay $R$. Node $N_{1}$ transmits data to node $N_{2}$ and at the same time node $N_{2}$ is also communicating data to $N_{1}$ through relay $R . N_{1}$ and $N_{2}$ transmit over the same channel. 

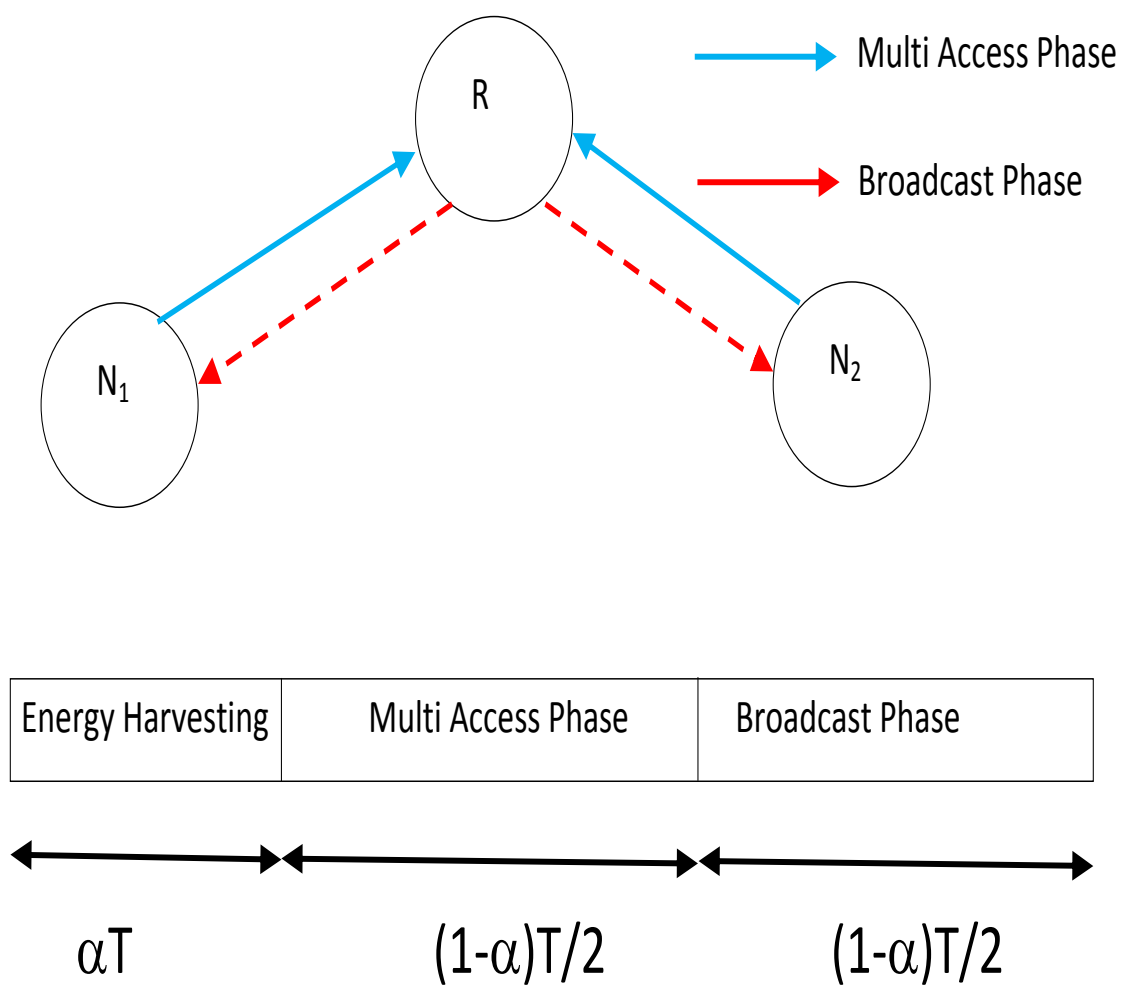

Figure 1: Two way relaying with Energy harvesting.

The frame with duration $T$ is decomposed in three parts :

- The first slot with duration $\alpha T$ is dedicated to energy harvesting. Relay $R$ harvests energy from RF signal transmitted by nodes $N_{1}$ and $N_{2}$.

The harvested energy is written as

$$
E=\beta\left(P_{1}\left|h_{N_{1} R}\right|^{2}+P_{2}\left|h_{N_{2} R}\right|^{2}\right) \alpha T=\beta\left(E_{1}\left|h_{N_{1} R}\right|^{2}+E_{2}\left|h_{N_{2} R}\right|^{2}\right) \alpha p,
$$

where $0<\alpha<1$ is harvesting duration percentage, $P_{i}$ (resp. $E_{i}$ ) is the transmit power (resp. symbol energy) of node $N_{i}$ and $h_{N_{1} R}$ (respectively $h_{N_{2} R}$ ) is channel coefficient between nodes $N_{1}$ (respectively $N_{2}$ ) and $R . p=T / T_{s}$ is the number of symbols per frame $T$. We have $E_{X}=T_{s} P_{X}$

- During the second time slot with duration $(1-\alpha) T / 2, N_{1}$ and $N_{2}$ transmit data to node $R$ over the same channel. This is the multiple access phase. The received signal at $R$ is written as

$$
y_{R}(j)=\sqrt{E_{1}} x_{1}(j) h_{N_{1} R}+\sqrt{E_{2}} x_{2}(j) h_{N_{2} R}+n_{R}(j)
$$

where $E_{i}$ is the transmitted energy per symbol of node $i$ with $1 \leq i \leq 2, x_{i}(j)$ is the $j$-th transmitted symbol by node $N_{i}$ and $n_{R}(j)$ is an Addivite White Gaussian Noise (AWGN) 
with variance $N_{0}$. A Rayleigh block fading channel is assumed where the channel remains constant over all the time frame with duration $T$.

- During the third time slot with duration $(1-\alpha) T / 2, R$ transmits amplifies the received signal to nodes $N_{1}$ and $N_{2}$. This is the broadcast phase.

Relay $R$ uses the harvested energy $E$ to amplify the received signal $y_{R}(j)$ to $N_{1}$ and $N_{2}$. The transmit symbol energy of $R$ is equal to the harvested energy $E$ devided by the number of transmitted symbols during $(1-\alpha) T / 2$ seconds i.e. $(1-\alpha) T /\left(2 T_{s}\right)=(1-\alpha) p / 2$ with $p=T_{s} / T$ :

$$
\begin{aligned}
E_{R} & =\frac{E}{(1-\alpha) p / 2}=\frac{\beta\left(E_{1}\left|h_{N_{1} R}\right|^{2}+E_{2}\left|h_{N_{2} R}\right|^{2}\right) \alpha p}{(1-\alpha) p / 2} \\
& =2 \frac{\alpha \beta}{(1-\alpha)}\left(E_{1}\left|h_{N_{1} R}\right|^{2}+E_{2}\left|h_{N_{2} R}\right|^{2}\right)
\end{aligned}
$$

Using (43), the amplification factor $G$ used by relay $R$ is written as

$$
G=\sqrt{\frac{E_{R}}{E_{1}\left|h_{N_{1} R}\right|^{2}+E_{2}\left|h_{N_{2} R}\right|^{2}+N_{0}}}
$$

\subsection{SNR at node $\mathrm{N}_{1}$}

The received signal at $N_{1}$ is written as

$$
y_{1}(j)=G h_{R N_{1}} y_{R}(j)+n_{1}(j),
$$

where $n_{1}(j)$ is an AWGN with variance $N_{0}$.

Using (43), we deduce

$$
\begin{aligned}
y_{1}(j)= & G h_{R N_{1}}\left[\sqrt{E_{1}} x_{1}(j) h_{N_{1} R}+\sqrt{E_{2}} x_{2}(j) h_{N_{2} R}+n_{R}(j)\right]+n_{1}(j), \\
= & \sqrt{E_{1}} G h_{R N_{1}} x_{1}(j) h_{N_{1} R}+\sqrt{E_{2}} G h_{R N_{1}} x_{2}(j) h_{N_{2} R} \\
& +G h_{R N_{1}} n_{R}(j)+n_{1}(j) .
\end{aligned}
$$

Node $N_{1}$ removes the self interference, $\sqrt{E_{1}} G h_{R N_{1}} x_{1}(j) h_{N_{1} R}$, since it knows the value of symbol $x_{1}(j)$. After removing self interference, we obtain

$$
y_{1}(j)=\sqrt{E_{2}} G h_{R N_{1}} x_{2}(j) h_{N_{2} R}+G h_{R N_{1}} n_{R}(j)+n_{1}(j) .
$$

The SNR at $N_{1}$ is written as

$$
\Gamma_{1}=\frac{E_{2} G^{2}\left|h_{R N_{1}}\right|^{2}\left|h_{N_{2} R}\right|^{2}}{N_{0}+N_{0} G^{2}\left|h_{R N_{1}}\right|^{2}} .
$$

Using the expression of amplification factor $G$ (45), we deduce

$$
\Gamma_{1}=\frac{E_{2}\left|h_{R N_{1}}\right|^{2}\left|h_{N_{2} R}\right|^{2}}{\frac{N_{0}}{G^{2}}+N_{0}\left|h_{R N_{1}}\right|^{2}}=\frac{\left|h_{R N_{1}}\right|^{2} E_{2}\left|h_{N_{2} R}\right|^{2}}{\frac{N_{0}}{E_{R}}\left[N_{0}+E_{1}\left|h_{N_{1} R}\right|^{2}+E_{2}\left|h_{N_{2} R}\right|^{2}\right]+N_{0}\left|h_{R N_{1}}\right|^{2}} .
$$


We assume that channels are reciprocal i.e. $h_{N_{1} R}=h_{R N_{1}}$. By neglecting the term in $N_{0}^{2}$ and using (44), the SNR at node $N_{1}$ lower bounded by

$$
\Gamma_{1}>\frac{2 \frac{\alpha \beta}{N_{0}(1-\alpha)} E_{2}\left|h_{R N_{1}}\right|^{2}\left|h_{N_{2} R}\right|^{2}}{1+2 \frac{\alpha \beta}{(1-\alpha)}\left|h_{R N_{1}}\right|^{2}}
$$

This upper bound is tight at high average SNR as the term $N_{0}^{2}$ can be neglected. We can write

$$
\Gamma_{1}>\frac{a_{1} X_{1} X_{2}}{1+a_{2} X_{1}}
$$

where

$$
\begin{gathered}
a_{1}=2 \frac{\alpha \beta}{N_{0}(1-\alpha)} E_{2}, \\
a_{2}=2 \frac{\alpha \beta}{(1-\alpha)}, \\
X_{1}=\left|h_{R N_{1}}\right|^{2},
\end{gathered}
$$

and

$$
X_{2}=\left|h_{N_{2} R}\right|^{2} .
$$

\section{$2.2 \quad \mathrm{SNR}$ at node $\mathrm{N}_{2}$}

The received signal at $N_{2}$ is written as

$$
y_{2}(j)=G h_{R N_{2}} y_{R}(j)+n_{2}(j),
$$

where $n_{2}(j)$ is an AWGN with variance $N_{0}$.

Using (43), we deduce

$$
\begin{aligned}
y_{2}(j)= & G h_{R N_{2}}\left[\sqrt{E_{1}} x_{1}(j) h_{N_{1} R}+\sqrt{E_{2}} x_{2}(j) h_{N_{2} R}+n_{R}(j)\right]+n_{2}(j), \\
= & \sqrt{E_{1}} G h_{R N_{2}} x_{1}(j) h_{N_{1} R}+\sqrt{E_{2}} G h_{R N_{2}} x_{2}(j) h_{N_{2} R} \\
& +G h_{R N_{2}} n_{R}(j)+n_{2}(j) .
\end{aligned}
$$

Node $N_{2}$ removes the self interference, $\sqrt{E_{2}} G h_{R N_{2}} x_{2}(j) h_{N_{2} R}$, since it knows the value of symbol $x_{2}(j)$. After removing self interference, we obtain

$$
y_{2}(j)=\sqrt{E_{1}} G h_{R N_{2}} x_{1}(j) h_{N_{1} R}+G h_{R N_{2}} n_{R}(j)+n_{2}(j) .
$$

The SNR at $N_{2}$ is written as

$$
\Gamma_{2}=\frac{E_{1} G^{2}\left|h_{R N_{2}}\right|^{2}\left|h_{N_{1} R}\right|^{2}}{N_{0}+N_{0} G^{2}\left|h_{R N_{2}}\right|^{2}} .
$$


Using the expression of amplification factor $G(45)$, we deduce

$$
\Gamma_{2}=\frac{E_{1}\left|h_{R N_{2}}\right|^{2}\left|h_{N_{1} R}\right|^{2}}{\frac{N_{0}}{G^{2}}+N_{0}\left|h_{R N_{2}}\right|^{2}}=\frac{\left|h_{R N_{2}}\right|^{2} E_{1}\left|h_{N_{1} R}\right|^{2}}{\frac{N_{0}}{E_{R}}\left[N_{0}+E_{1}\left|h_{N_{1} R}\right|^{2}+E_{2}\left|h_{N_{2} R}\right|^{2}\right]+N_{0}\left|h_{R N_{2}}\right|^{2}} .
$$

We assume that channels are reciprocal i.e. $h_{N_{2} R}=h_{R N_{2}}$. By neglecting the term in $N_{0}^{2}$ and using (44), the SNR at node $N_{1}$ lower bounded by

$$
\Gamma_{2}>\Gamma_{2}^{\text {low }}=\frac{2 \frac{\alpha \beta}{N_{0}(1-\alpha)} E_{1}\left|h_{R N_{2}}\right|^{2}\left|h_{N_{1} R}\right|^{2}}{1+2 \frac{\alpha \beta}{(1-\alpha)}\left|h_{R N_{2}}\right|^{2}}
$$

This upper bound is tight at high average SNR as the term $N_{0}^{2}$ can be neglected.

We can write

$$
\Gamma_{2}^{\text {low }}=\frac{a_{1} X_{1} X_{2}}{1+a_{2} X_{2}}
$$

where

$$
\begin{gathered}
a_{1}=2 \frac{\alpha \beta}{N_{0}(1-\alpha)} E_{2} \\
a_{2}=2 \frac{\alpha \beta}{(1-\alpha)} \\
X_{1}=\left|h_{R N_{1}}\right|^{2}
\end{gathered}
$$

and

$$
X_{2}=\left|h_{N_{2} R}\right|^{2}
$$

\subsection{Two way relaying in the presence of multiple relays}

Fig. 2 shows the principle of TWR in the presence of K relays. The selected relay offers the largest SNR at node $N_{1}$ or $N_{2}$. When the selected relay maximizes the SNR at node $N_{1}$, the CDF of SNR is the products of CDF of SNRs of different relays

$$
F_{\Gamma_{1}}(x)=\prod_{k=1}^{K} F_{\Gamma_{1}^{k}}(x)
$$

where $\Gamma_{1}^{k}$ is the SNR at node $N_{1}$ when relay $R_{k}$ is the active relay. $\Gamma_{1}^{k}$ is given in (9). 


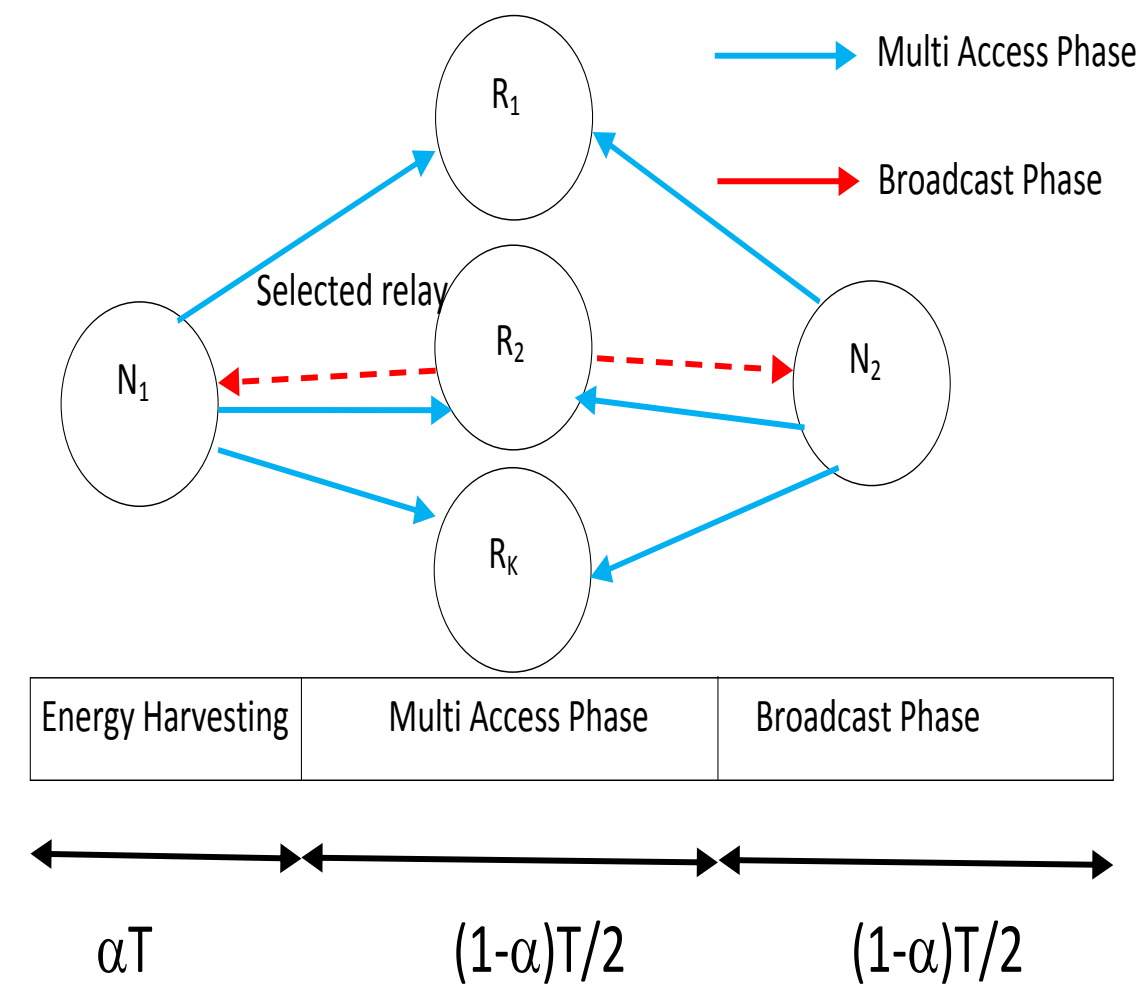

Figure 2: Two way relaying with Energy harvesting in the presence of $K$ relays.

\section{CDF of SNR}

\subsection{CDF of SNR at node $\mathrm{N}_{1}$}

The SNR at node $N_{1}$ is lower bounded by

$$
\Gamma_{1}>\Gamma_{1}^{\text {low }}=\frac{a_{1} X_{1} X_{2}}{1+a_{2} X_{1}} .
$$

The CDF of SNR is upper bounded by

$$
F_{\Gamma_{1}}(x)<F_{\Gamma_{1}^{l o w}}(x)=P\left(\Gamma_{1}^{\text {low }} \leq x\right) .
$$

We have

$$
\begin{aligned}
P\left(\Gamma_{1}^{\text {low }}\right. & \leq x)=\int_{0}^{+\infty}\left[1-P\left(\Gamma_{1}^{\text {low }}>x \mid X_{1}=u\right)\right] f_{X_{1}}(u) d u \\
& =\int_{0}^{+\infty}\left[1-P\left(\frac{a_{1} u X_{2}}{1+a_{2} u}>x\right)\right] f_{X_{1}}(u) d u
\end{aligned}
$$


where $f_{X_{1}}(u)$ is the Probability Density Function (PDF) of $X_{1}$.

For Rayliegh fading channels, $X_{1}$ is exponentially distributed with mean

$$
\frac{1}{\lambda_{1}}=E\left(X_{1}\right)=E\left(\left|h_{R N_{1}}\right|^{2}\right) .
$$

We deduce

$$
\begin{aligned}
P\left(\Gamma_{1}^{\text {low }}\right. & \leq x)=\int_{0}^{+\infty}\left[1-P\left(X_{2}>\frac{x\left(1+a_{2} u\right)}{a_{1} u}\right)\right] \lambda_{1} e^{-u \lambda_{1}} d u \\
& =\int_{0}^{+\infty}\left[1-e^{-\frac{x\left(1+a_{2} u\right) \lambda_{2}}{a_{1} u}}\right] \lambda_{1} e^{-u \lambda_{1}} d u
\end{aligned}
$$

We use the following result grad

$$
\int_{0}^{+\infty} e^{-\frac{b}{4 v}-a v} d v=\sqrt{\frac{b}{a}} K_{1}(a b),
$$

where $K_{1}(x)$ is the modified Bessel function of first order and second kind.

We finally obtain

$$
F_{\Gamma_{1}}(x)<P\left(\Gamma_{1}^{l o w} \leq x\right)=1-2 \lambda_{1} e^{-\frac{a_{2} x \lambda_{2}}{a_{1}}} \sqrt{\lambda_{2} \frac{x}{a_{1} \lambda_{1}}} K_{1}\left(2 \sqrt{\lambda_{1} \frac{x \lambda_{2}}{a_{1}}}\right) .
$$

\subsection{CDF of SNR at node $\mathrm{N}_{2}$}

The SNR at node $N_{2}$ is lower bounded by

$$
\Gamma_{2}>\Gamma_{2}^{\text {low }}=\frac{a_{1} X_{1} X_{2}}{1+a_{2} X_{2}} .
$$

The CDF of SNR is upper bounded by

$$
F_{\Gamma_{2}}(x)<F_{\Gamma_{2}^{l o w}}(x)=P\left(\Gamma_{2}^{l o w} \leq x\right) .
$$

We have

$$
\begin{aligned}
P\left(\Gamma_{2}^{\text {low }}\right. & \leq x)=\int_{0}^{+\infty}\left[1-P\left(\Gamma_{2}^{\text {low }}>x \mid X_{2}=u\right)\right] f_{X_{2}}(u) d u \\
& =\int_{0}^{+\infty}\left[1-P\left(\frac{a_{1} X_{1} u}{1+a_{2} u}>x\right)\right] f_{X_{2}}(u) d u
\end{aligned}
$$

We deduce

$$
\begin{aligned}
P\left(\Gamma_{2}^{\text {low }}\right. & \leq x)=\int_{0}^{+\infty}\left[1-P\left(X_{1}>\frac{x\left(1+a_{2} u\right)}{a_{1} u}\right)\right] \lambda_{2} e^{-u \lambda_{2}} d u \\
& =\int_{0}^{+\infty}\left[1-e^{-\lambda_{1} \frac{x\left(1+a_{2} u\right)}{a_{1} u}}\right] \lambda_{2} e^{-u \lambda_{2}} d u
\end{aligned}
$$


We use (34), to deduce

$$
F_{\Gamma_{2}}(x)<P\left(\Gamma_{2}^{\text {low }} \leq x\right)=1-2 \lambda_{2} e^{-\frac{a_{2} x \lambda_{1}}{a_{1}}} \sqrt{\lambda_{1} \frac{x}{a_{1} \lambda_{2}}} K_{1}\left(2 \sqrt{\lambda_{1} \frac{x \lambda_{2}}{a_{1}}}\right) .
$$

\section{PEP}

In this section, we derive the expression of the average Packet Error Probability (PEP). The PEP can be tightly upper bounded by [21]

$$
\mathrm{PEP} \leq \int_{0}^{w_{0}} f_{\Gamma}(\gamma) d \gamma
$$

where $f_{\Gamma}(\gamma)$ is the Probability Density Function (PDF) of SNR $\Gamma$ and $w_{0}$ is a waterfall threshold.

Equation (13) shows that the PEP for a given instantaneous SNR, $\gamma \leq w_{0}$, can be approximated to 1 . However, the PEP for a given instantaneous SNR, $\gamma>w_{0}$ can be approximated to $0[21]$.

Hence,

$$
\mathrm{PEP} \leq F_{\Gamma}\left(w_{0}\right),
$$

where $F_{\Gamma}(x)$ is the Cumulative Distribution Function (CDF) of the received SNR. We denote $\bar{\Gamma}=\frac{E_{b}}{N_{0}}$ as the average SNR, where $E_{b}$ is the transmitted energy per bit, $N_{0}$ is the noise Power Spectral Density (PSD) and $w_{0}$ is a waterfall threshold written as [21],

$$
w_{0}=\int_{0}^{+\infty} g(\gamma) d \gamma
$$

$g(\gamma)$ is the PEP for a given instantaneous SNR, $\gamma=\bar{\Gamma}|h|^{2}$ and $h$ is the channel coefficient.

\subsection{PEP for uncoded transmission}

For uncoded $M$-QAM modulation, we have

$$
g(\gamma)=1-\left(1-P_{e s}(\gamma)\right)^{\frac{N+n_{d}}{\log _{2}(M)}}
$$

where $N$ is the number of useful information bits per packet, $n_{d}$ is the number of parity bits per packet and $P_{e s}$ is the Symbol Error Probability (SEP) given as [22]

$$
P_{e s}(\gamma) \simeq 2\left(1-\frac{1}{\sqrt{M}}\right) \operatorname{erfc}\left(\sqrt{\frac{\log _{2}(M) 3 \gamma}{(M-1) 2}}\right) .
$$

$\operatorname{erfc}(x)$ is the complementary error function,

$$
\operatorname{erfc}(x) \leq e^{-x^{2}}
$$

Using (45) and (46), the SEP is approximated by 


$$
P_{e s} \simeq a_{1} e^{-c_{1} \gamma}
$$

where,

$$
\begin{gathered}
a_{1}=2\left(1-\frac{1}{\sqrt{M}}\right), \\
c_{1}=\frac{3 \log _{2}(M)}{2(M-1)}
\end{gathered}
$$

\subsection{PEP with Channel Coding}

If a convolutional encoding is used, $g(\gamma)$ can expressed as,

$$
g(\gamma)=1-\left(1-P_{E}(\gamma)\right)^{\frac{N+n_{d}}{\log _{2}(M)}}
$$

where

$$
P_{E}(\gamma) \leq \sum_{d=d_{f}}^{+\infty} a_{d} P_{d}(\gamma)
$$

$d_{f}$ and $a_{d}$ are respectively the free distance and the number of trellis with Hamming weight $d$. Further,

$$
P_{d}(\gamma) \simeq 2\left(1-\frac{1}{\sqrt{M}}\right) \operatorname{erfc}\left(\sqrt{\frac{3 R_{c} d \gamma \log _{2}(M)}{2(M-1)}}\right) .
$$

where $R_{c}$ is the rate of convolutional encoding.

Using the approximation in (46) and keeping only the first term of (23), we have

$$
P_{E}(\gamma) \simeq a_{2} e^{-c_{2} \gamma}
$$

where

$$
\begin{gathered}
a_{2}=a_{d_{f}} 2\left(1-\frac{1}{\sqrt{M}}\right), \\
c_{2}=\frac{3 R_{c} d_{f} \log _{2}(M)}{2(M-1)} .
\end{gathered}
$$

Hence, we can generalize $g(\gamma)$ as follow,

$$
g(\gamma) \simeq 1-\left(1-a_{i} e^{-c_{i} \gamma}\right)^{\frac{N+n_{d}}{\log _{2}(M)}},
$$

where $i=1$ in the absence of any channel coding and $i=2$ for convolutional coding. 


\subsection{Waterfall Threshold}

Using (43), the waterfall threshold is given by

$$
w_{0} \simeq k_{1} \ln \left(\frac{N+n_{d}}{\log _{2}(M)}\right)+k_{2}
$$

where the Proof is provided in Appendix A.

$$
\begin{gathered}
k_{1}=\frac{1}{c_{i}}, \\
k_{2}=\frac{E+\ln \left(a_{i}\right)}{c_{i}},
\end{gathered}
$$

$E \simeq 0.577$ is the Euler constant.

\section{Optimal Packet Length for TWR}

The average number of attempts of HARQ protocols is equal to

$$
T_{r}=\sum_{i=1}^{+\infty} P E P^{i-1}(1-P E P)=\frac{1}{1-P E P}
$$

Therefore, the throughput in bit/s/Hz is expressed as

$$
\begin{aligned}
T h r & =\frac{\log _{2}(M) N}{\left(N+n_{d}\right) T_{s} B T_{r}}=\frac{\log _{2}(M) N}{\left(N+n_{d}\right)}(1-P E P) \\
& \geq \frac{\log _{2}(M) N}{\left(N+n_{d}\right)}\left[1-F_{\Gamma}\left(w_{0}\right)\right]
\end{aligned}
$$

where $B=1 / T_{s}$ is the used bandwidth and $T_{s}$ is the symbol period.

The optimal packet length maximizing the throughput can be obtained using the Gradient algorithm.

$$
N(i+1)=N(i)+\mu \frac{\partial T h r(N=N(i))}{\partial N}
$$

We can write

$$
\frac{\partial T h r}{\partial N}=\frac{\log _{2}(M) n d}{\left(N+n_{d}\right)^{2}}\left[1-F_{\Gamma}\left(w_{0}\right)\right]-\frac{\log _{2}(M) N}{\left(N+n_{d}\right)} f_{\Gamma}\left(w_{0}\right) \frac{k_{1}}{N+n_{d}}
$$

OPL can be applied to maximize the throughput at node $N_{1}$ or $N_{2}$. 


\section{$6 \quad$ Theoretical and simulation results}

Simulation results were obtained using MATLAB as a simulation environment.

Simulation results were performed by measuring the Packet Error Rate (PER) to deduce the throughput. The packet error rate is the number of erroneous packets/number of transmitted packets. We made simulation until 1000 packets are erroneously received.

Fig. 3 and 4 show the throughput at node $N_{1}$ for $\alpha=1 / 3$, a QPSK modulation for average SNR 10 and $20 \mathrm{~dB}$. The distance between all nodes is equal to 1 . We notice that we can maximize the throughput by choosing the packet length. Also, the throughput increases as the number of relays increase due to cooperative diversity. In fact, we always select the relay with the largest SNR. Finally, by comparing Fig. 3 and 4, we observe that packet length should be increased as the average SNR increases. There is good accordance between theoretical and simulation results.

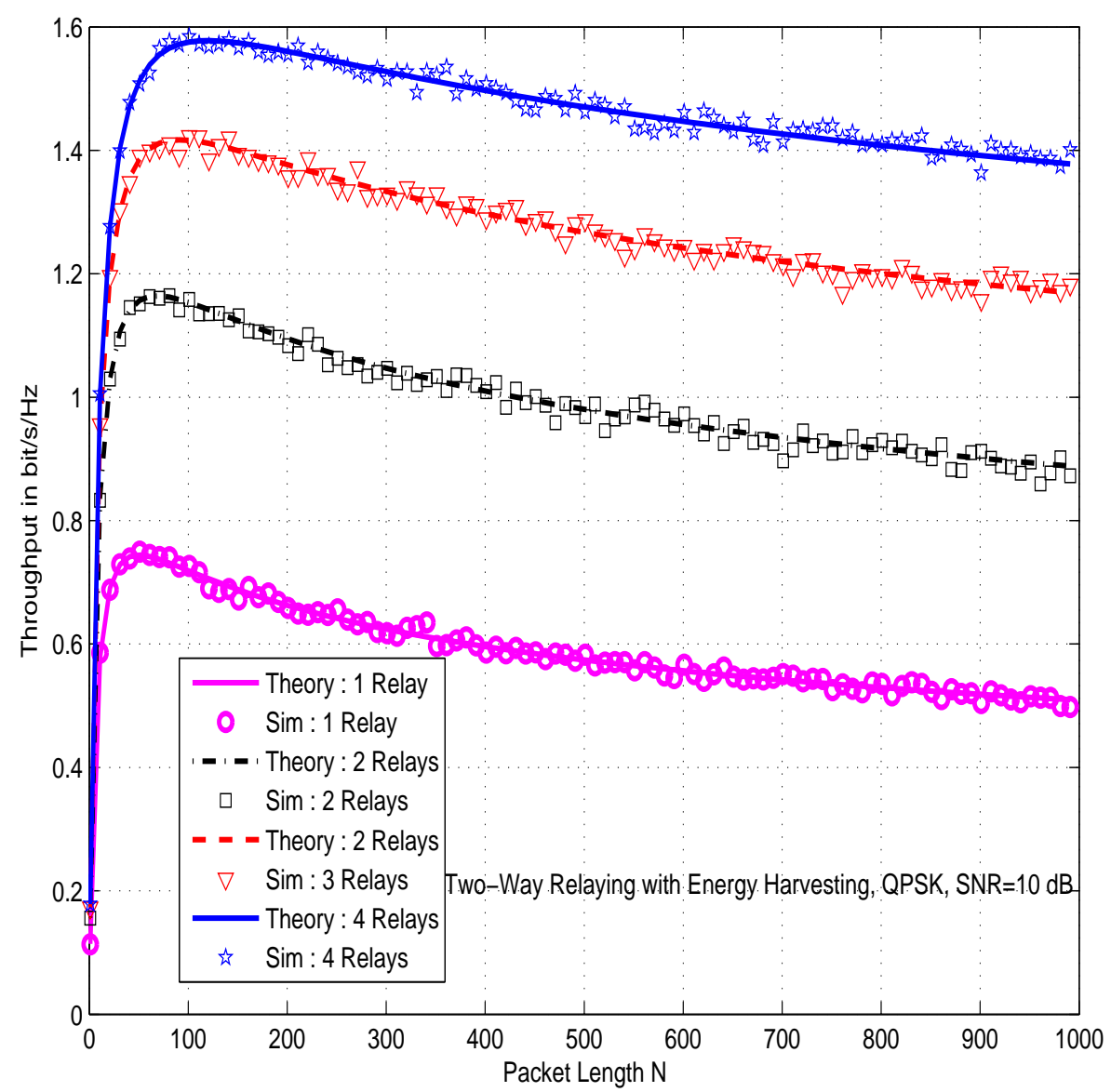

Figure 3: Throughput at node $N_{1}$ versus packet length at $\mathrm{SNR}=10 \mathrm{~dB}: 64$ QAM modualtion. 
International Journal of Computer Networks \& Communications (IJCNC) Vol.11, No.3, May 2019

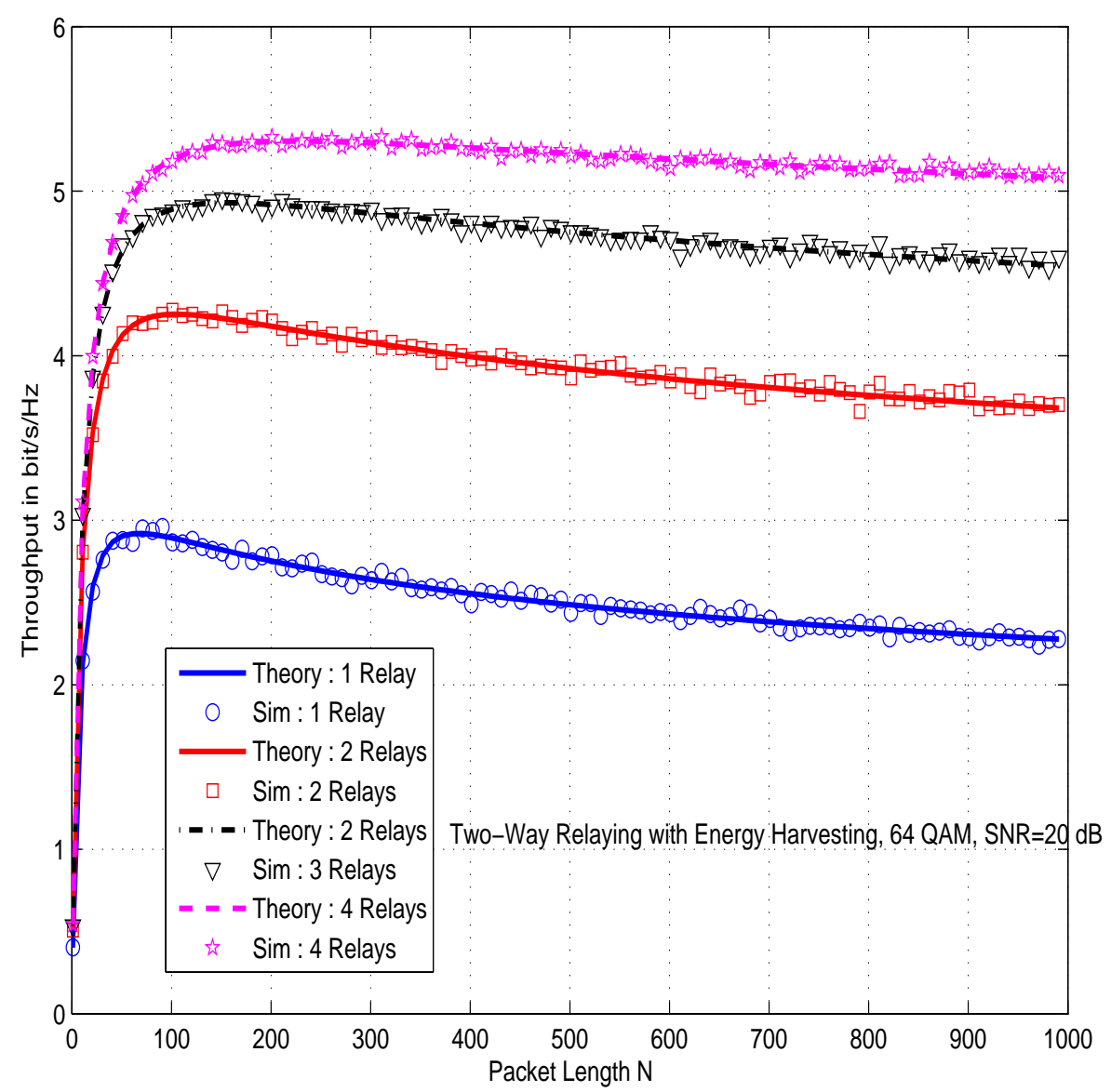

Figure 4: Throughput at node $N_{1}$ versus packet length at SNR=20 dB: 64 QAM modualtion.

Fig. 5 shows that OPL offers higher throughput than Fixed Packet Length (FPL) as studied in [1-20]. These results correspond to throughput of $N_{1}$ for $\alpha=1 / 3$. They were obtained using MATLAB for a 64 QAM modulation. In fact, the proposed optimal packet length allows maximizing the throughput. If the SNR is low, the packet length is decreased. However, at high SNR, we can increase packet length. 


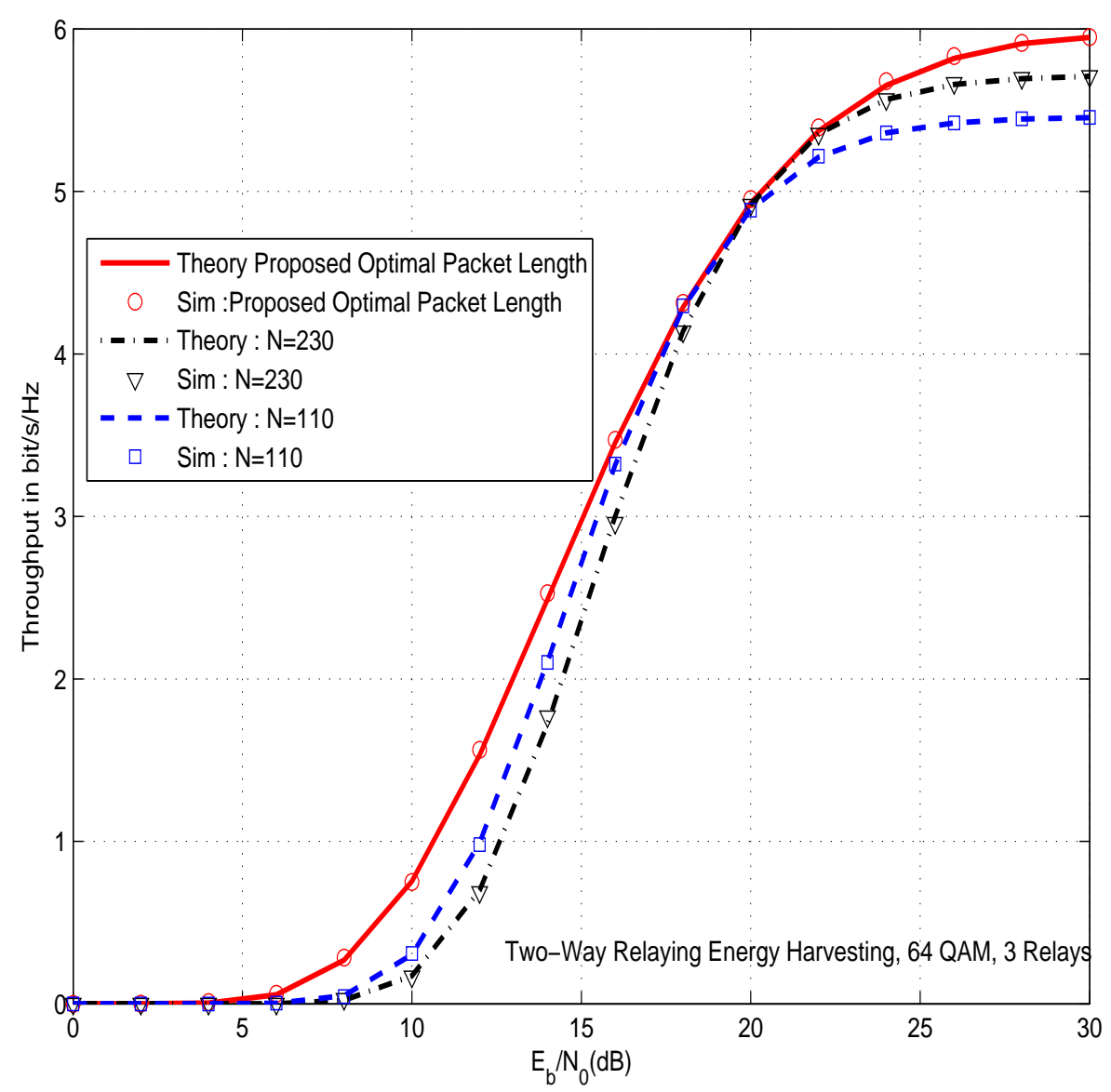

Figure 5: Throughput at node $N_{1}$ for OPL and FPL :64 QAM modualtion.

Fig. 6 shows the OPL for QPSK, 16 QAM and 64 QAM modulation. We observe that packet length should be increases when we use a small modulation such as QPSK. When 64 QAM modulation is used, packet length should be reduced since the PEP is high. Also packet length should be increased (respectively decreased) at high (respectively low) SNR. 


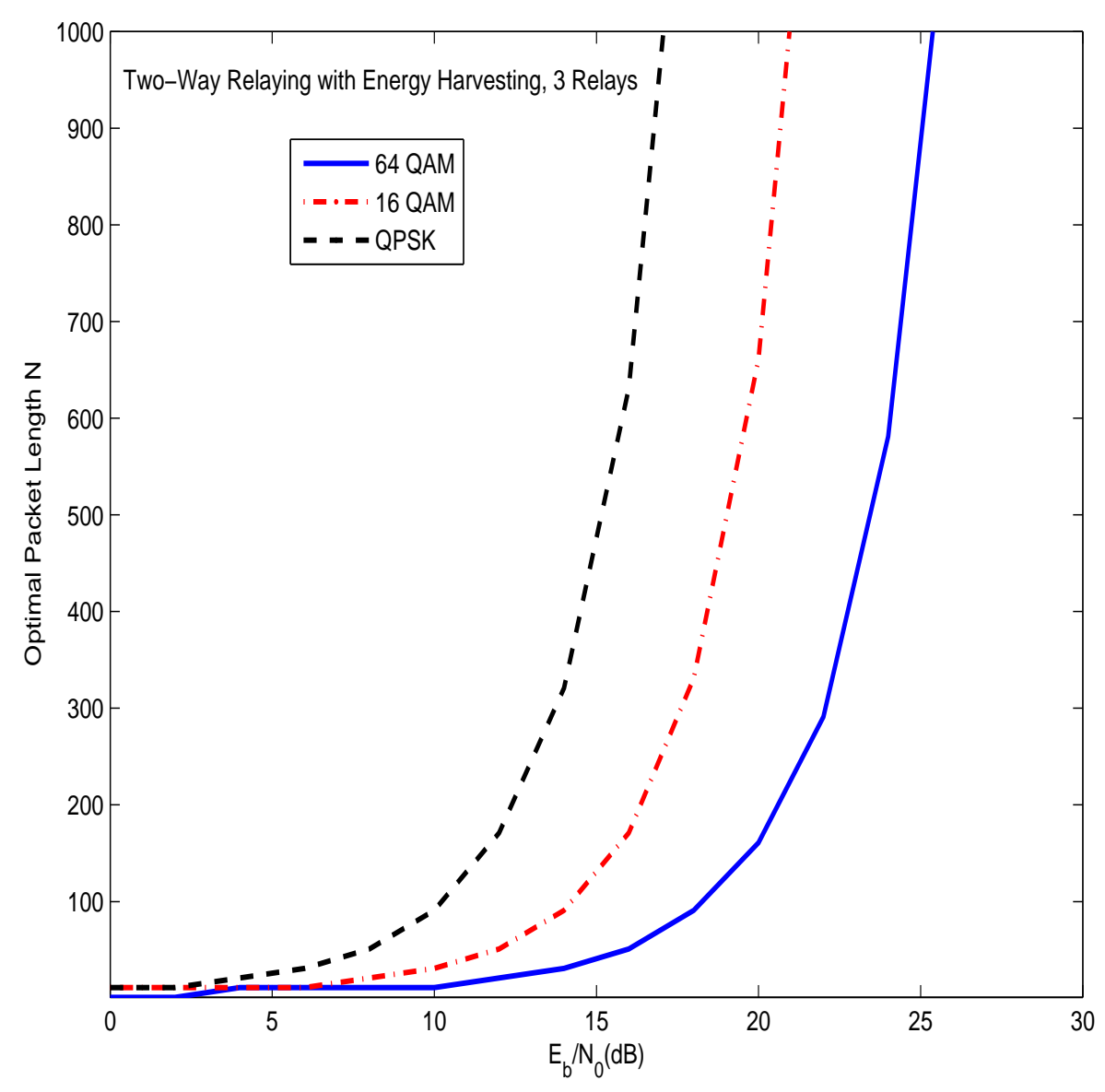

Figure 6: Optimal packet length versus SNR.

\section{Conclusion}

In this paper, we suggested enhancing the throughput of Two Way Relaying (TWR) with energy harvesting. We derive the best packet length that yields the largest throughput at node $N_{1}$ or $N_{2}$. Our study is valid for energy harvesting systems where the relay harvest energy from RF signals transmitted by source nodes $N_{1}$ and $N_{2}$. We have shown that the proposed TWR with best packet length offers better throughput than previous studies. Also, the throughput can be enhanced by increasing the number of relays. The proposed optimal packet length can be used in Wireless Sensor Networks (WSN) with two way relaying. 
International Journal of Computer Networks \& Communications (IJCNC) Vol.11, No.3, May 2019

Appendix A : We have

$$
w_{0}=\int_{0}^{+\infty}\left[1-\left(1-a_{i} e^{-c_{i} u}\right)^{\frac{N+n_{d}}{\log _{2}(M)}}\right] d u
$$

We deduce

$$
w_{0}=\frac{1}{c_{i}} \int_{0}^{a_{i}}\left[1-(1-y)^{\left.\frac{N+n_{d}}{\log _{2}(M)}\right]} \frac{d y}{y}\right.
$$

Therefore, we have

$$
w_{0}=\frac{1}{c_{i}} \int_{1-a_{i}}^{1} \frac{1}{1-x}\left[1-x^{\frac{N+n_{d}}{\log _{2}(M)}}\right] d x
$$

We obtain

$$
w_{0}=\frac{1}{c_{i}} \int_{1-a_{i}}^{1} \sum_{k=0}^{\frac{N+n_{d}}{\log _{2}(M)}-1} x^{k} d x
$$

We deduce

$$
w_{0}=\frac{1}{c_{i}} \sum_{k=1}^{\frac{N+n_{d}}{\log _{2}(M)}}\left(\frac{1}{k}-\frac{\left(1-a_{i}\right)^{k}}{k}\right) .
$$

For $\frac{N+n_{d}}{\log _{2}(M)}>>1$, we can write

$$
\sum_{k=1}^{\frac{N+n_{d}}{\log _{2}(M)}} \frac{1}{k}=\ln \left(\frac{N+n_{d}}{\log _{2}(M)}\right)+E
$$

and

$$
\sum_{k=1}^{\frac{N+n_{d}}{\log _{2}(M)}} \frac{\left(1-a_{i}\right)^{k}}{k} \simeq \sum_{k=1}^{+\infty} \frac{\left(1-a_{i}\right)^{k}}{k}=-\ln \left(a_{i}\right) .
$$

Combining (68), (69) and (70), we obtain (57).

\section{References}

[1] Mahdi Attaran ; Jacek Ilow, "Signal Alignment in MIMO Y Channels with Twoway Relaying and Unicast Traffic Patterns", 2018 11th International Symposium on Communication Systems, Networks and Digital Signal Processing (CSNDSP), Year: 2018, Page s: 1 - 6 .

[2] B. Dutta ; R. Budhiraja ; R. D. Koilpillai ; L. Hanzo, "Analysis of Quantized MRCMRT Precoder For FDD Massive MIMO Two-Way AF Relaying", IEEE Transactions on Communications, Year: 2018, ( Early Access ), Pages: 1 - 1. 
[3] Guo Li ; Feng-Kui Gong ; Hang Zhang ; Xiang Chen, "Non-coherent transmission for two-way relaying systems with relay having large-scale antennas", IET Communications, Year: 2018 , Volume: 12 , Issue: 16, Page s: 1991 - 1996.

[4] Jingon Joung, "Energy Efficient SpaceTime Line Coded Regenerative Two-Way Relay Under Per-Antenna Power Constraints", IEEE Access, Year: 2018 , Volume: 6, Pages: 47026 - 47035.

[5] Fabien Heliot ; Rahim Tafazolli, "Energy-Efficient Sources and Relay Precoding Design for Two-Way Two-Hop MIMO-AF Systems", 2018 European Conference on Networks and Communications (EuCNC), Year: 2018, Page s: 87 - 92, IEEE Conferences.

[6] Xiaolong Lan ; Qingchun Chen ; Xiaohu Tang ; Lin Cai, "Achievable Rate Region of the Buffer-Aided Two-Way Energy Harvesting Relay Network", IEEE Transactions on Vehicular Technology, Year: 2018, Volume: 67, Issue: 11, Pages: 11127 - 11142.

[7] Sucharita Chakraborty, Debarati Sen, "Iterative SAGE-Based Joint MCFOs and Channel Estimation for Full-Duplex Two-Way Multi-Relay Systems in Highly Mobile Environment", IEEE Transactions on Wireless Communications, Year: 2018 , Volume: 17 , Issue: 11, Pages: 7379 - 7394.

[8] Ugrasen Singh ; Sourabh Solanki ; Devendra S. Gurjar ; Prabhat K. Upadhyay ; Daniel B. da Costa, "Wireless Power Transfer in Two-Way AF Relaying with Maximal-Ratio Combining under Nakagami-m Fading", 14th International Wireless Communications and Mobile Computing Conference (IWCMC) Year: 2018, Pages: $169-173$.

[9] Kisong Lee ; Jun-Pyo Hong ; Hyun-Ho Choi ; Tony Q. S. Quek, "Wireless-Powered Two-Way Relaying Protocols for Optimizing Physical Layer Security", IEEE Transactions on Information Forensics and Security,

[10] Xinghua Jia ; Chaozhu Zhang ; Il-Min Kim, "Optimizing Wireless Powered Two-Way Communication System With EH Relays and Non-EH Relays", Year: 2019 , Volume: 14, Issue: 1, IEEE Transactions on Vehicular Technology, Pages: 162 - 174. Year: 2018 , Volume: 67 , Issue: 11, Pages: 11248 - 11252.

[11] Mehdi Ashraphijuo ; Morteza Ashraphijuo ; Xiaodong Wang, "On the DoF of TwoWay MIMO Relay Networks", IEEE Transactions on Vehicular Technology, Year: 2018 , Volume: 67 , Issue: 11, Page s: 10554 - 10563.

[12] Sanaz Ghorbani ; Ali Jamshidi ; Alireza Keshavarz-Haddad, "Performance Evaluation of Joint Relay Selection and Network Coding in Two-Way Relaying Wireless Communication Networks", Iranian Conference on Electrical Engineering (ICEE), Year: 2018, Page s: $755-757$.

[13] Ali H. Bastami ; Abolqasem Hesam, "Network-Coded Cooperative Spatial Multiplexing in Two-Way Relay Channels", IEEE Transactions on Vehicular Technology, Year: 2018 , Volume: 67 , Issue: 11, Pages: 10715 - 10729. 
[14] Shiqi Gong ; Chengwen Xing ; Shaodan Ma ; Zhongshan Zhang ; Zesong Fei, "Secure Wideband Beamforming Design for Two-Way MIMO Relaying Systems", IEEE Transactions on Vehicular Technology, Year: 2018, ( Early Access ), Pages: 1 - 1.

[15] Tamer Mekkawy ; Rugui Yao ; Nan Qi ; Yanan Lu, "Secure Relay Selection for Two Way Amplify-and-Forward Untrusted Relaying Networks", IEEE Transactions on Vehicular Technology, Year: 2018, ( Early Access ) Pages: 1 - 1.

[16] Mahendra K. Shukla ; Suneel Yadav ; Neetesh Purohit, "Secure Transmission in Cellular Multiuser Two-Way Amplify-and-Forward Relay Networks", IEEE Transactions on Vehicular Technology, Year: 2018, ( Early Access ) Page s: 1 - 1.

[17] Hongbin Xu ; Li Sun, "Encryption Over the Air: Securing Two-Way Untrusted Relaying Systems Through Constellation Overlapping", IEEE Transactions on Wireless Communications, Year: 2018 , ( Early Access ) Page s: $1-1$.

[18] Ruifeng Gao ; Xiaodong Ji ; Ye Li ; Yingdong Hu ; Zhihua Bao, "Secure Power Allocation of Two-Way Relaying with an Untrusted Denoise-and-Forward Relay", 2018 15th International Symposium on Wireless Communication Systems (ISWCS), Year: 2018, Pages: 1 - 5.

[19] Mohanad Obeed ; Wessam Mesbah, "Improving Physical Layer Security in Two-Way Relay Systems", 2018 25th International Conference on Telecommunications (ICT), Year: 2018, Pages: 220 - 224.

[20] Chensi Zhang ; Jianhua Ge ; Fengkui Gong ; Yancheng Ji ; Jinxi Li, "Improving Physical-Layer Security for Wireless Communication Systems Using Duality-Aware Two-Way Relay Cooperation", IEEE Systems Journal, Year: 2018 , Pages: 1 - 9.

[21] Y. Xi, A. Burr, J. B. Wei, D. Grace, " A general upper bound to evaluate packet error rate over quasi-static fading channels", IEEE Trans. Wireless Communications, vol. 10, nO 5, pp 1373-1377, May 2011.

[22] J. Proakis, "Digital Communications", Mac Graw-Hill, 5th edition, 2007. 